\title{
Projeto de aterramento para sistema monofilar com retorno pelo terra ${ }^{1}$
}

\author{
H umberto C. Bertolo², D elly O liveira Filho², Tarcísio A. Pizziolo², Denilson E. Rodrigues², \\ Paulo M. B. Monteiro ${ }^{2} \&$ G ustavo A. Xavier ${ }^{2}$
}

\begin{abstract}
RESU MO
Este trabalho apresenta o projeto de aterramento elétrico para Sistema M onofilar com Retorno pela Terra (MRT), em solicitações de baixas frequências, utilizando-se o método das imagens e considerando a simplificação do método dos potenciais constantes. Simulou-se o comportamento de três diferentes configurações de aterramento: hastes em triângulo, em quadrado vazio e para nove hastes, formando uma malha com quatro quadrados. A partir da simulação obtiveram-se o valor da resistência de aterramento para cada configuração e os potenciais gerados na superfície do solo. Com base nesses resultados foi possível comparar os valores tabelados para resistências mínimas, em função da potência do transformador, aplicados para diferentes concessionárias de energia que utilizam o Sistema MRT, com os valores calculados que mantêm os potenciais no solo em níveis seguros para seres humanos e animais. Os resultados mostraram que é possível utilizar aterramentos mais simples e mais baratos, pois os gradientes de tensões no solo se mantiveram em níveis seguros.
\end{abstract}

Palavras-chave: MRT, eletrificação rural, comportamento do aterramento

\section{Grounding project for single wire earth return system}

\begin{abstract}
This work presents a grounding project for a Single Wire Earth Return (SWER) system in low frequency applications, through the use of the image method, a simplification of the constant potential method. The responses of three different grounding configurations were simulated: electrodes in triangular format, electrodes placed in the corners of a square and nine electrodes placed in the corners of a grid with four squares. With the mentioned simulation it was possible to calculate the ground resistance and the surface voltage of each configuration. Based on these results, it was possible to compare the values for minimal resistance, depending on the power of the transformer, applied to various power utilities that use the SW ER system with the values that hold the potential in the soil at safe levels for human beings and animals. Results showed that it is possible to use simpler and cheaper grounding configurations due to the fact that the surface voltages were maintained at safe levels.
\end{abstract}

Key words: SWER, rural electrification, grounding behavior

\footnotetext{
${ }^{1}$ Extraído da Dissertação de Mestrado apresentada pelo primeiro autor à U niversidade Federal de Viçosa, U FV, para obtenção do título Magister Scientiae do programa de pós-graduação em Engenharia Agrícola

2 UFV Av. P.H. Rolfs, s/ n, Campus Universitário - CEP 36570-000, Viçosa, M G. Fone: (31) 3899-1897; (31) 3899-1233; (31) 3899-4082; (31) 3899-1897; (31) 3899-1896; (31) 3899-1930. E-mail: humberto.calil@power.alstom.com; delly@ufv.br; pizziolo@ufv.br; deduardo@ufv.br; paulo@em.ufop.br; gustavo.xavier@ufv.br
} 


\section{INTRODUÇÃO}

Nas últimas décadas tem-se observado, no Brasil, um crescimento considerável dos índices de eletrificação rural (Oliveira Filho et al., 2005). Porém, como a instalação das redes de distribuição de energia elétrica rural tem sido confiada às concessionárias, ela é tratada como extensão dos serviços prestados nas cidades utilizando-se, muitas vezes, sistemas de eletrificação rural trifásicos, postes de concreto e outros materiais e dispositivos que a encarecem (Haanyika, 2006).

Na maioria das vezes não é necessário utilizar alimentadores trifásicos para a eletrificação das zonas rurais. Devido à grande importância da energia elétrica no campo, o padrão exigido pelas concessionárias de postes de concreto e de sistemas trifásicos ou monofásicos tradicionais, é questionável. Podese, por exemplo, utilizar postes de madeira e Sistemas Monofilar com Retorno por Terra (MRT), para diminuição de custos (Haanyika, 2008; Kashem \& Ledwich, 2004).

Como substituição aos alimentadores trifásicos tem-se os monofásicos, com custos menores. Com um custo menor ainda em relação aos alimentadores trifásicos, tem-se o sistema monofilar com retorno por terra. Os custos dos sistemas bifásico, monofásico, MRT com cabos de alumínio e MRT com cabo de aço zincado, são de 80, 52, 50 e $27 \%$ dos custos do trifásico, respectivamente (CEPEL, 2005; Owen, 1998).

O sistema monofilar com retorno por terra exige que se tenha um aterramento de baixa resistência e, para tal, pode-se usar um sistema de aterramento em malhas. No projeto de malhas de aterramento em baixa frequência deve-se considerar o aspecto de desempenho e a segurança relacionada ao valor da resistência de aterramento e com o potencial na superfície do solo (Pereira Filho \& Cardoso, 2001; Liu et al., 2005).

Em sistemas MRT o meio de dispersão da corrente de retorno é o solo. Como o solo pode apresentar variações em função da forma geológica, nível de compactação, teor de umidade e sais nele contidos, um bom projeto de aterramento é essencial para garantir a utilização segura deste sistema (Caminha Júnior, 2001; He et al., 2003).

As ligações à terra devem ser estáveis e de resistência adequada pois as redes monofilares têm seu desempenho, tanto sob o aspecto de confiabilidade como de segurança, estritamente vinculado às condições dos seus aterramentos. Além disso, um rompimento nas conexões do aterramento gera tensões perigosas (Brooking et al., 1992; Zeng et al., 2005; Chen et al., 2004).

As normas técnicas de algumas companhias de energia elétrica recomendam um valor mínimo de resistência de aterramento, de forma que o gradiente de tensão no aterramento se limite a $27 \mathrm{~V}$ impedindo, assim, potenciais de passo e de toque que prejudiquem a segurança de pessoas e animais (CEPEL, 2005).

Em sistemas de aterramento cada eletrodo, individualmente, e o efeito combinado de todos eles, determinam a resistência de aterramento do sistema (Garrett \& Pruitt, 1985; Tuma et al., 2006).

Neste trabalho o objetivo foi desenvolver um programa computacional capaz de calcular a resistência de aterramento e os potenciais no solo para as configurações mais comuns de sistemas de aterramento. Por meio dos resultados obtidos é possível prever se os potenciais no solo, gerados por diferentes condições de funcionamento de sistemas MRT, serão prejudiciais aos homens e animais.

\section{MATERIAL E MÉTODOS}

Neste trabalho se utilizou o método das imagens juntamente com o método dos potenciais constantes, para calcular os potenciais no solo e a resistência de aterramento de diversas configurações para sistemas MRT, a qual foi obtida determinando-se a resistência transversal, ou seja, a resistência imposta para a corrente, que se dissipa para o solo.

Para a modelagem de um sistema de aterramento composto de várias hastes verticais, determina-se o potencial gerado em uma haste, i, devido à corrente que circula em outra haste, j. A resistência entre essas duas hastes, $\mathrm{R}_{\mathrm{ij}}$, é definida pelo potencial médio causado na haste i dividido pela corrente dispersa pela haste j, de acordo com a Eq. 1:

$$
R i j=\frac{\rho}{4 \pi L_{j} L_{i}} \int_{L_{j}} \int_{L_{i}} \frac{1}{r} d_{L_{i}} d L_{j}
$$

na qual: $\mathrm{L}_{\mathrm{i}}$ é o comprimento do eletrodo $\mathrm{i}(\mathrm{m}), \mathrm{L}_{\mathrm{j}}$ é o comprimento do eletrodo j (m), $\rho$ é a resistividade do solo $(\Omega \mathrm{m})$ e r é a distância de um ponto $\mathrm{P}$ qualquer a um elemento infinitesimal da haste (m).

Para se determinar as resistências de aterramento, os efeitos das imagens dos segmentos das hastes por onde circula a corrente devem ser somados.

O método das imagens consiste na conversão de um campo elétrico em outro mais simples de se manipular. Este método permite reduzir o problema original a um conjunto de imagens que reproduzem o potencial da fonte em determinado eixo, conforme a Eq. 2:

$$
\left[\begin{array}{c}
\mathrm{V}_{1} \\
\vdots \\
\mathrm{V}_{\mathrm{i}}
\end{array}\right]=\left[\begin{array}{ccc}
\mathrm{R}_{11} & \cdots & \mathrm{R}_{1 \mathrm{j}} \\
\vdots & \ddots & \vdots \\
\mathrm{R}_{\mathrm{i} 1} & \cdots & \mathrm{R}_{\mathrm{ij}}
\end{array}\right]\left[\begin{array}{c}
\mathrm{I}_{1} \\
\vdots \\
\mathrm{I}_{\mathrm{j}}
\end{array}\right]
$$

Por meio da solução da Eq. 2 e se considerando $\mathrm{V}_{1}=\mathrm{V}_{2}=\ldots=\mathrm{V}_{\mathrm{i}}$ e a corrente injetada no aterramento $\mathrm{I}_{\mathrm{TOTAL}}=\mathrm{I}_{1}+\mathrm{I}_{2}+\ldots+\mathrm{I}_{\mathrm{j}}$, obtémse a resistência total do sistema de aterramento.

Os potenciais são obtidos utilizando-se a Eq. 3.

$$
\mathrm{Vp}=\frac{\rho}{4 \pi \mathrm{L}} \int_{0}^{\mathrm{L}} \frac{\mathrm{I}_{\mathrm{TOTAL}}}{\mathrm{r}} \mathrm{dl}
$$

Desenvolveu-se um programa computacional capaz de estimar a resistência de aterramento e os potenciais no solo de sistemas de aterramento com diferentes configurações, em função da variação dos seguintes parâmetros: potência do 
transformador, tensão de distribuição, resistividade do solo, comprimento, diâmetro, profundidade e espaçamento das hastes de aterramento, resistência de aterramento e potenciais no solo.

Uma modelagem computacional foi feita para se determinar o efeito de diferentes parâmetros do sistema MRT nos valores obtidos para o sistema de aterramento.

A Figura 1 indica ofluxograma do programa.

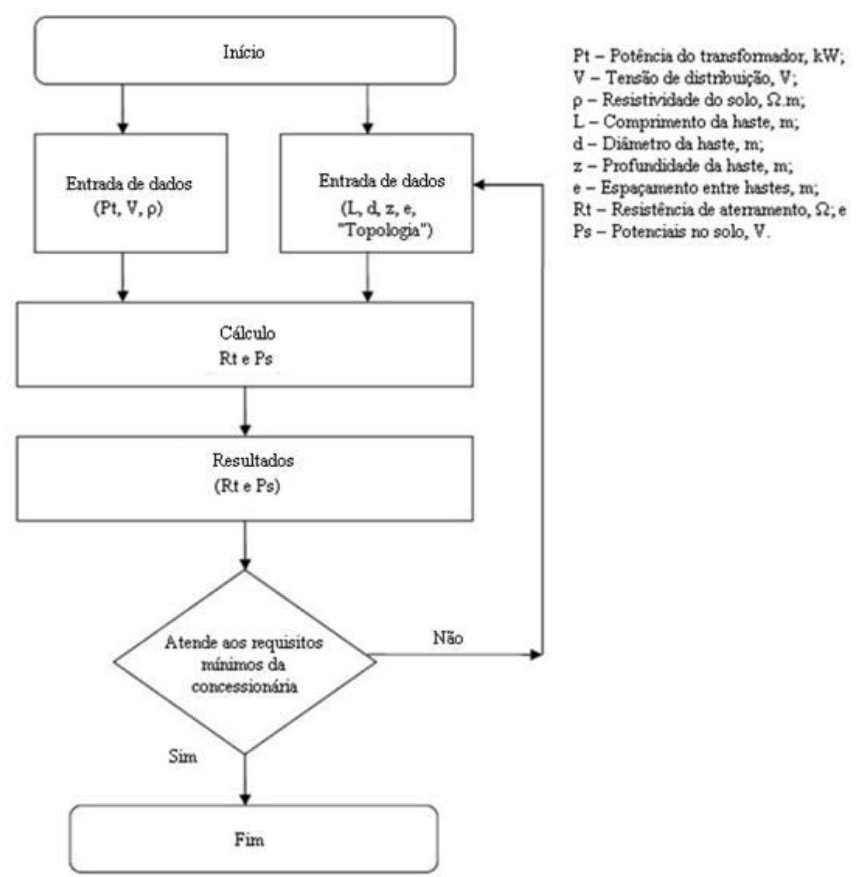

Figura 1. Fluxograma para o projeto do aterramento

Para a simulação, os seguintes parâmetros devem ser especificados:

- Potência do transformador de isolamento e tensão de distribuição do sistema MRT. Por meio desses valores será computada a corrente injetada no sistema de aterramento

- A resistividade do solo

- O comprimento, o diâmetro, a profundidade e o espaçamento entre as hastes

- Tipo de topologia de aterramento. O programa oferece quatro topologias: uma haste, três hastes em triângulo, quatro hastes em um quadrado e nove hastes, formando uma malha com quatro quadrados

As três topologias estudadas neste trabalho podem ser vistas na Figura 2

A partir dos dados de entrada o programa calcula a resistência equivalente e os gradientes de potencial no solo, por meio da metodologia descrita em Pereira Filho \& Cardoso (2001).

Devido à dificuldade de se obter analiticamente as soluções das integrais duplas, elas foram calculadas numericamente, pelo método de Simpson.

Os dados de saída apresentados ao usuário são a resistência total do sistema de aterramento, a distribuição do potencial elétrico ao longo de uma linha partindo do centro da malha e o potencial de passo máximo tolerável para humanos e animais.
A.

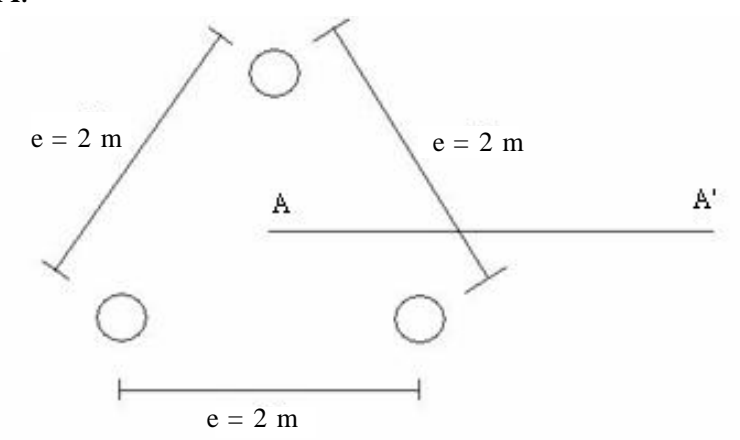

B.

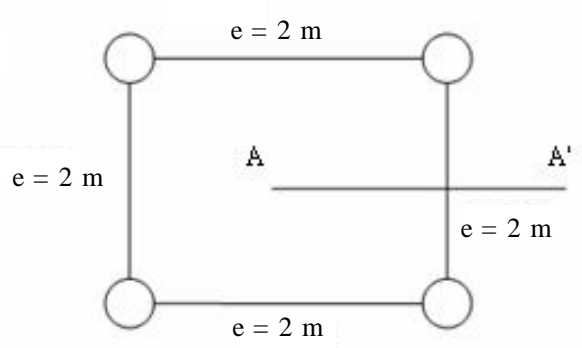

C.

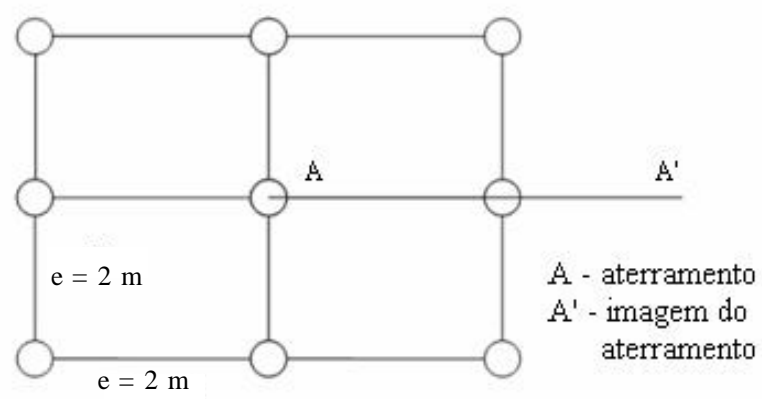

Figura 2. Configurações de aterramento: Configuração em triângulo do aterramento com três hastes $(A)$; configuração em quadrado do aterramento, com quatro hastes (B); configuração em malha do aterramento com nove hastes formando quatro quadrados (C)

Para se analisar o potencial de passo em humanos, considerou-se a distância de um metro entre os pés, conforme descrito por norma; em animais, consideraram-se dois metros.

\section{RESULTADOS E DISCUSSÃO}

Os resultados abaixo foram obtidos para hastes com 0,0125 $\mathrm{m}$ diâmetro, 2,4 $\mathrm{m}$ de comprimento, 0,5 $\mathrm{m}$ de profundidade e com espaçamento de $2 \mathrm{~m}$. A tensão primária é de 13,8 por raiz quadrada de $3 \mathrm{kV}$ e a resistividade do solo foi considerada constante, com valor de 100 Ùm, porém se encontram resistividades do solo que variam de 100 a $700 \Omega \mathrm{m}$. O valor da resistividade do solo influencia, de forma muito significativa, o potencial de passo estudado neste trabalho.

Por meio da resistência de aterramento e da diferença máxima de potencial no solo, é possível determinar se o sistema atende às necessidades mínimas de proteção contra os gradientes de 
potencial gerados pela circulação de corrente pela terra e aos valores máximos de resistência de aterramento para transformadores de distribuição propostos pela Guia Rural (1988) e pelas concessionárias de energia que utilizam o sistema MRT, mostrados na Tabela 1.

Tabela 1. Valores máximos de resistência de terra $(\Omega)$ para transformadores de distribuição conectados na tensão 13,8 por raiz quadrada de $3 \mathrm{kV}$

\begin{tabular}{cccccc}
\hline \multirow{2}{*}{ Empresa } & \multicolumn{7}{c}{ Transformadores (kVA) } \\
\cline { 2 - 6 } & $\mathbf{3}$ & $\mathbf{5}$ & $\mathbf{1 0}$ & $\mathbf{1 5}$ & $\mathbf{2 5}$ \\
CEMAR & 20 & 20,0 & 20,0 & - & - \\
CELPE & 65 & 40,0 & 20,0 & 15,0 & - \\
COBER & 30 & 30,0 & 20,0 & 13,3 & - \\
LIGHT & - & 42,5 & 21,2 & 14,2 & - \\
CELESC & - & 10,0 & 10,0 & 10,0 & 10,0 \\
CEEE & - & 20,0 & 20,0 & 20,0 & 10,0 \\
ELETROBRÁS & 71 & 42,5 & 21,2 & 14,2 & 8,5 \\
\hline
\end{tabular}

Fonte: (CEPEL, 2005)

Pode-se criticar a adoção de valores fixos de resistência de aterramento, visto que:

a. Pode-se onerar desnecessariamente o sistema

b. O nível de segurança no uso de transformadores de diferentes potências não é constante, sendo maior nos transformadores menores

c. A falta de padronização impõe dificuldades técnicas à integração de sistemas de diferentes concessionárias

\section{Análise das topologias}

O sistema de três hastes em triângulo é mostrado na Figura 2A. A resistência de aterramento obtida para este sistema com os valores dos parâmetros descritos acima, foi $\mathrm{R}_{\mathrm{t} 3}=17,37 \Omega$. Este valor atende aos parâmetros da Guia Rural (1988) para transformadores com potências de 3,5 e $10 \mathrm{kVA}$, porém, de acordo com a Tabela 1, este valor é alto para transformadores de isolamento com potências entre 15 e $25 \mathrm{kVA}$.

A topologia com quatro hastes é mostrada na Figura 2B. A resistência de aterramento obtida para este sistema considerando-se os valores dos parâmetros descritos acima, foi $\mathrm{R}_{\mathrm{t} 4}=14,24 \Omega$, o que atende aos valores mínimos prescritos pela Guia Rural (1988) para transformadores com potências de $3,5,10$ e $15 \mathrm{kVA}$.

A configuração com nove hastes formando uma malha com 4 quadrados é mostrada na Figura 2C. A resistência de aterramento obtida para este sistema, considerando-se os valores dos parâmetros, foi $R_{t 9}=8,00 \Omega$. Este valor atende à indicação da tabela da Guia Rural para todas as potências dos transformadores de isolamento.

Os valores máximos de resistência de terra em função da potência do transformador, também foram calculados por Caminha Júnior (2001) e são muito próximos aos valores máximos propostos pela Guia Rural (1988).

A diferença entre os valores calculados para as três topologias está de acordo com Caminha Júnior (2001), que afirma que a geometria do posicionamento e a quantidade dos eletrodos é um fator determinante para a diminuição da resistência de aterramento.
Os potenciais no solo obtidos para os diferentes valores de potências do transformador de isolamento e topologias estão representados nas Figuras 3A, 3B e 3C. Esses valores estão referenciados ao centro do sistema de aterramento, distanciando-se dele ao longo de uma linha reta, conforme a Figura 2.

A.

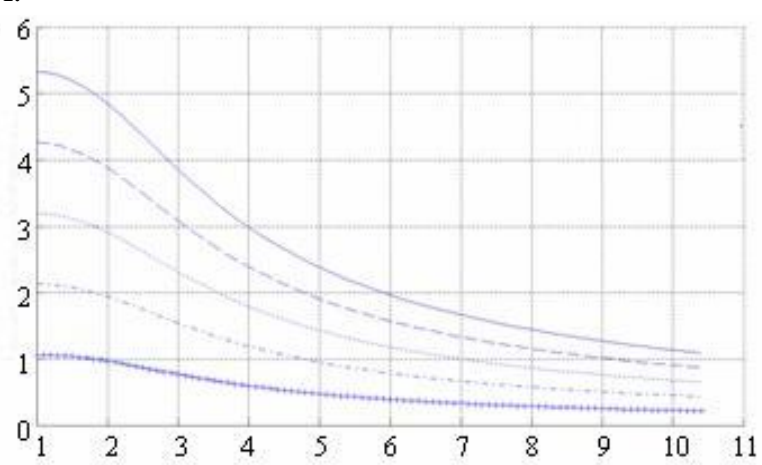

B.

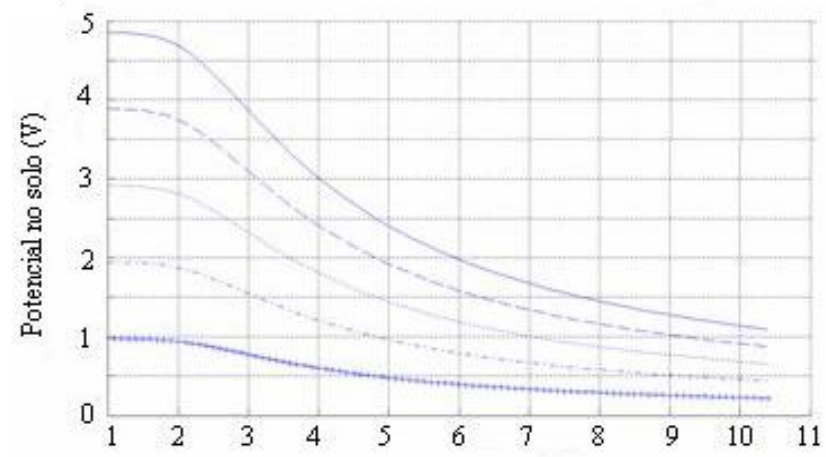

C.

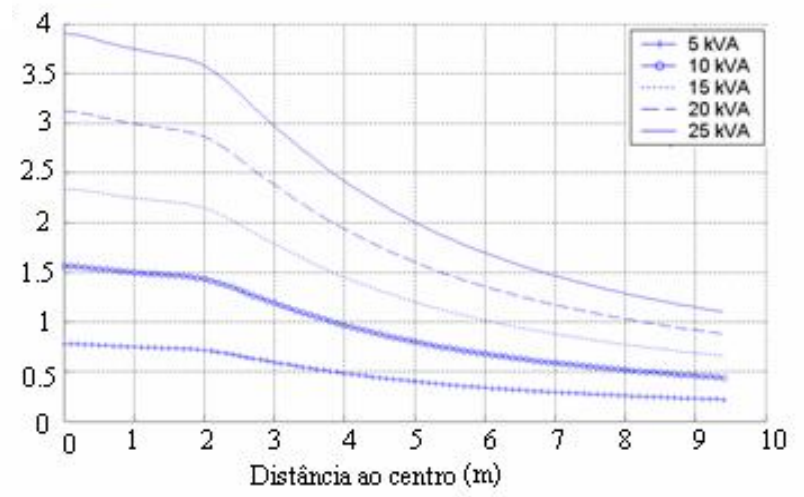

Figura 3. Potenciais gerados no solo: Sistema de aterramento com três hastes em triângulo (A); sistema de aterramento com quatro hastes formando um quadrado (B); si stema de aterramento com nove hastes formando uma malha com quatro quadrados (C) para resistividade do solo de $100 \Omega \mathrm{m}$

Comparando os gráficos da Figura 3 com a curva de potencial mostrada por Caminha Júnior (2001), pode-se verificar que os valores calculados estão de acordo com o comportamento real de um sistema de aterramento.

Por meio das Figuras 3A e 3B, verifica-se que, a um metro do centro, o potencial no solo possui o valor máximo nas 
Tabela 2. Tensões de passo para humanos e animais para diferentes valores de potência do transformador de isolamento, utilizando-se aterramento com hastes em triângulo, quadrado e mal ha

\begin{tabular}{|c|c|c|c|c|c|c|}
\hline \multirow{2}{*}{$\begin{array}{c}\text { Potência do } \\
\text { transformador (kVA) }\end{array}$} & \multicolumn{3}{|c|}{ Tensão de passo (1 m) máxima em humanos (V) } & \multicolumn{3}{|c|}{ Tensão de passo ( $2 \mathrm{~m}$ ) máxima em animais (V) } \\
\hline & Triângulo & Quadrado & Malha & Triângulo & Quadrado & Malha \\
\hline 5 & 0,20 & 0,18 & 0,13 & 0,37 & 0,34 & 0,23 \\
\hline 10 & 0,40 & 0,37 & 0,25 & 0,74 & 0,67 & 0,46 \\
\hline 15 & 0,60 & 0,55 & 0,38 & 1,12 & 1,02 & 0,70 \\
\hline 20 & 0,80 & 0,73 & 0,51 & 1,49 & 1,35 & 0,93 \\
\hline 25 & 1,00 & 0,92 & 0,63 & 1,87 & 1,69 & 1,16 \\
\hline
\end{tabular}

configurações com três e quatro hastes. À medida em que se afasta do centro do aterramento, o valor do potencial no solo diminui. Quanto maior é a potência do transformador maior também é a inclinação negativa da curva, resultando em uma diferença maior de tensão no solo. Os potenciais no solo tendem a zero quando se aumenta a distância ao centro da haste.

Verifica-se, por meio da Figura 3C que, a partir do centro do aterramento da topologia com nove hastes, o potencial no solo diminui. Quanto maior é a potência do transformador maior também é a inclinação negativa da curva, resultando em uma diferença maior de tensão no solo. Os potenciais no solo tendem a zero quando se aumenta muito a distância ao centro da haste.

As tensões de passo máximas encontradas para os diferentes valores de potência do transformador de isolamento para as topologias com três, quatro e nove hastes, estão representadas na Tabela 2.

Para comparação entre as três configurações de aterramento discutidas, gerou-se o gráfico da Figura 4, no qual podem ser vistos os potenciais gerados no solo pelas configurações de aterramento em triângulo, quadrado e em malha com nove hastes para a potência de $15 \mathrm{kVA}$ no transformador e resistividade do solo de $100 \Omega \mathrm{m}$. Os potenciais têm valores bem próximos nas configurações de três e quatro hastes. A configuração de nove hastes apresenta valores menores de potencial no solo que as outras duas configurações de

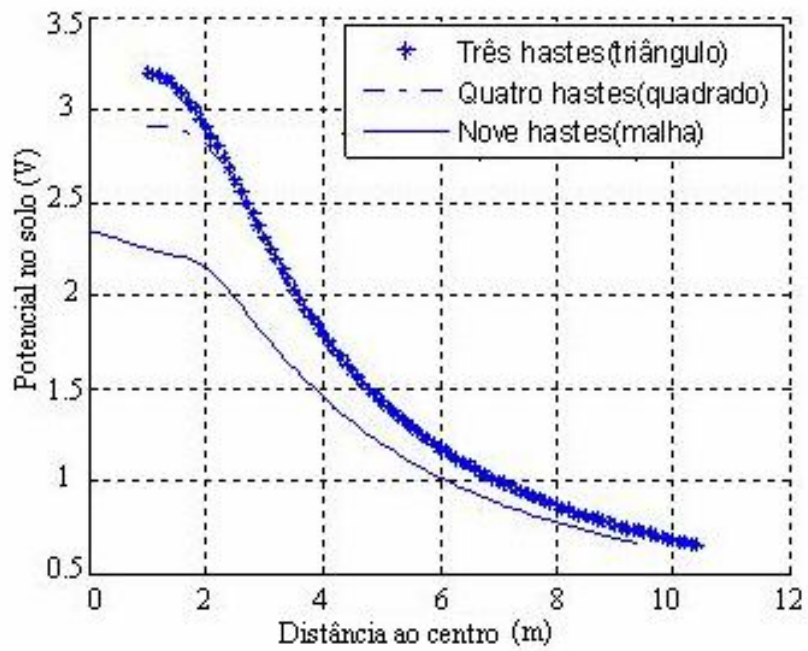

Figura 4. Comparação entre os potenciais gerados no solo para as três configurações de aterramento, com um transformador de 15 kVA e resistividade do solo de 100 $\Omega \mathrm{m}$ aterramento, qualquer que seja a distância ao centro. À medida em que a distância aumenta, os valores de potencial no solo gerado por cada uma das três configurações se aproximam e tendem a zero, visto que o potencial é inversamente proporcional à distância do centro.

Caminha Júnior (2001) realizou testes durante 3 anos com um transformador de $15 \mathrm{kVA}$, com um sistema de aterramento em triângulo mais um eletrodo adicional e mediu as tensões de passo, em distâncias de até $5 \mathrm{~m}$ ao redor do poste. Os valores medidos variaram entre 0,0014 e 1,5410 V, mudando de acordo com teor de umidade no solo, que influenciava também a resistividade do solo e o valor da resistência de aterramento.

Os cálculos das tensões de passo e pata neste trabalho foram feitos utilizando-se um valor fixo da resistividade do solo $100 \Omega \mathrm{m}$ e da resistência de aterramento, $(17,37 ; 14,24$; $8,00 \Omega$ ) para os sistemas de três, quatro e de nove hastes de aterramento, respectivamente, representando uma situação bem específica de condições do solo e de parâmetros físicos considerados.

\section{ConClusõES}

1. Elaborou-se um programa no qual é possível calcular a resistência de aterramento e os potenciais, em função da distância do aterramento em qualquer ponto do solo e para qualquer configuração de aterramento.

2. A única configuração simulada que resulta em uma resistência de aterramento que atende a todas as potências dos transformadores de isolamento, foi uma malha contendo nove hastes.

3. Para solicitações de regime permanente a tabela de valores máximos de resistência do solo fornecida pelas concessionárias de energia elétrica está superdimensionada considerando-se os gradientes de potencial no solo.

\section{LITERATURA CITADA}

Brooking, T. R.; Rensburg, N. J. van; Fourie, R. The improved utilization of existing rural networks with the use of intermediate voltage and single wire earth return systems. In: IEEE AFRICON, 3, 1992, Ezulwini Valley. Proceedings... Ezulwini Valley: AFRICON, 1992, p.228-234.

Caminha Júnior, I. C. Análise dos potenciais de superfície gerados no solo por um sistema monofilar com retorno por terra. Botucatu: UNESP, 2001. 115p. Dissertação Mestrado 
CEPEL - Centro de Pesquisas de Energia Elétrica - Seleção de Sistemas - MRT. Relatórios de Eletrificação Rural, 2005.

Chen, L.; Chen, J.; Liang, T.; Wang, W. A study of grounding resistance reduction agent using granulated blast furnace slag. IEEE Transactions on Power Delivery, v.19, p.973-978, 2004.

Garret, D. L.; Pruitt, J. G. Problems encountered with the average potential method of analyzing substation grounding systems. IEEE Transactions on Power Apparatus Systems, v.104, p.3586-3596, 1985.

Guia Rural. Manual técnico de eletrificação rural. Sistema monofilar com retorno por terra (MRT). São Paulo: Editora Abril, 1988, 66p.

Haanyika, C. M. Rural electrification policy and institutional linkages. Energy Policy, v.34, p.2977-2993, 2006.

Haanyika, C. M. Rural electrification in Zambia: A policy and institutional analysis. Energy Policy, v.36, p.1044-1058, 2008.

He, J.; Zeng, R.; Gao, Y.; Tu, Y.; Sun, W.; Zou, J.; Guan, Z. Seasonal influences on safety of substation grounding system. IEEE Transactions on Power Delivery, v.18, p.788795, 2003

Kashem, M. A.; Ledwich, G. Distributed generation as voltage support for single wire earth return systems. IEEE Transactions on Power Delivery, v.19, p.1002-1011, 2004.
Liu, J.; Southey, R. D.; Dawalibi, F. P. Application of advanced grounding design techniques to plant grounding systems. In: IEEE/PES Transmission and Distribution Conference and Exibition: Asia and Pacific, Dalian. Dalian: 2005, p.1-6.

Oliveira Filho, D.; Ferenc, C. H. R.; Teixeira, C. A.; Dias, G. P.; Milagres R. C.; Pontes, C. R. Uso de motores monofásicos acoplados mecanicamente em série, em irrigação por pivô central. Revista Brasileira de Engenharia Agrícola e Ambiental, v.9, p.139-144, 2005.

Owen, E. L. Rural electrification: The long struggle. Industry Applications Magazine, IEEE , v.4, p.6-17, 1998.

Pereira Filho, M. L.; Cardoso, J. R. Avaliação de desempenho de malhas de terra usando imagens complexas: uma nova abordagem. Controle e Automação, v.12, p.215-223, 2001.

Tuma, E. T.; Santos, R. O.; Oliveira, R. M. S.; Sousa Sobrinho, C. L. S. Análise do comportamento transitório dos parâmetros de sistemas de aterramento utilizando o método FDTD, Revista IEEE America Latina, v.4, p.55-61, 2006.

Zeng, R.; He, J.; Lee, J.; Chang, S.; Tu, Y.; Gao, Y.; Zou, J.; Guan, Z. Influence of Overhead Transmission Line on Grounding Impedance Measurement of Substation. IEEE Transactions on Power Delivery, v.20, p.1219-1225, 2005. 